\title{
The development of binocular discrimination in infants
}

\author{
CLAUDIO S. HUTZ and HAROLD P. BECHTOLDT \\ University of Iowa, Iowa City, Iowa 52242
}

\begin{abstract}
The ability of 8- to 13-week-old infants to discriminate between stereoscopic and nonstereoscopic stimuli under short habituation experiences was tested repeatedly until they were 24 weeks old or able to reach for real objects with direction of gaze to one of two adjacent displays of a shadow-caster as the criterion measure. Stereopsis was demonstrated at the final session in $83 \%$ of the subjects by a reaching response to a virtual image. All but 1 of the 26 subjects discriminated on every test between depth and no-depth stimuli with no age differential; the subjects generally preferred the no-depth stimulus. While stereopsis and reactions to novelty or to complexity are possibile explanations of shadow-caster discriminations, the significant reaching response results plus the consistent gaze preference strongly support the hypothesis that binocular functioning is available at 8 weeks of age.
\end{abstract}

Increased interest in early visual development is clearly evident in a wide range of recent publications dealing with the responses both of animal and of human infants to visual stimuli. Much of this recent work has dealt with the contrast sensitivity or acuity function at a given age, although studies of binocular functioning also are appearing. However, there are few if any studies of binocular functioning dealing with the change over a period of time in early infancy. The aim of the present study is to provide some of the needed evidence of the development of binocular functioning over the age range of 8 to 24 weeks using a combined longitudinal/crosssectional sample design.

Studies of binocular fixation in infants suggest that newborns are already able to make convergent eye movements under certain conditions (Slater \& Findlay, 1975) and that after the 2nd month of life they can reliably converge on moving targets (Aslin, 1977).

Some researchers claim that some newborns have stereopsis (Bower, Broughton, \& More, 1970) while others (Appel \& Campos, 1977; Atkinson \& Braddick, 1976) report some eight-week-old infants provide evidence of stereoscopic discrimination. Fox, Aslin, Shea, and Dumais (1980) had an observer judge from an infant's direction of gaze and head movements the direction in which a dynamic random dot stereogram figure was moving; they report that 14 -week-old infants have stereopsis but that 10 -week-old infants do not. Unambiguous evidence of stereopsis is provided by the Fox et al. random dot stereogram at 14 weeks of age, as well as by the shadow-caster technique, in which a differential reaching response is utilized at about 20 weeks

This experiment constituted a portion of the requirements for the MA degree by C. S. Hutz under the supervision of H. P. Bechtoldt. The financial assistance of the graduate college of the University of Iowa for the payment of parents and for computing costs is gratefully acknowledged. of age (Bechtoldt \& Hutz, 1979; Gordon \& Yonas, 1976; Yonas, Oberg, \& Norcia, 1978). Held (Note 1) reports clear evidence of stereopsis in 10-week-old infants, obtained from a forced-choice procedure involving contoured stimuli.

The wide range of ages from the 1st week to the 20th week postpartum for the estimate of the appearance of stereopsis in human infants appears to be largely a function of the procedure used, including the nature of the test stimuli and the sample of subjects. A major problem leading to the wide range of estimated ages appears to be the testing procedure used. While the methods using the optokinetic and evoked potential response have considerable appeal, the preferential forced-choice procedure pioneered by Fantz (1956, 1958) has been widely accepted as a standard method (Dobson \& Teller, 1978; Giviazda, Brill, \& Held, 1979; Held, 1979). A possibly critical feature of the stimuli used is the presence or absence of monocular cues, the absence of which is achieved by the random dot stereograms. But the use of contoured stimuli certainly should not be ruled out, since there exists some evidence from adult subjects that different mechanisms may be involved in the processing of random dot stimuli and of stimuli with monocular contours (Benton \& Hecaen, 1970; Carmon \& Bechtoldt, 1969; Hamsher, 1978; Richards, 1977).

The preferential forced-choice method based on the shadow-caster technique (Gregory, 1964; Lee, 1969) has some special interpretative problems not found with standard stereogram forced-choice techniques; in addition, as Yonas and Pick (1975) point out, it is often not possible to identify the effective stimulus variables on the basis of differential fixation in the preferential choice techniques. The special problem with the preference procedure and the shadow-caster is the presence of differential monocular cues in addition to the retinal 
disparity cues; these monocular cues may influence the direction of fixation and the preference on the basis of complexity or novelty of pattern. As long as the percent of choice deviates markedly from $50 \%$ in a two-choice procedure, regardless of the choice, discrimination between the two choices is indicated. The absence of the control conditions of zero and vertical disparity in the shadow-caster procedure can be corrected by the use of a depth-indicator response like directed reaching, a response not available at 8 or 10 weeks of age. However, if the longitudinal observations are continued until 24 weeks of age, the procedure will provide a final control or "validation" test that, if successful, rules out the presence of monocular subjects, as well as the use of monocular complexity cues as the basis for the discrimination.

A measure of the relative effectiveness of the static retinal disparity or other binocular cues influencing the choice of patterns can be provided by also utilizing movement cues in separate forced-choice trials, since moving stimuli are known to be very effective stimuli at a very early age (Reese \& Lipsitt, 1970).

The choice of the shadow-caster procedure with one observer aware of the stimulus conditions was made in order to be able to test the infants in their homes. The testing of infants at home apparently reduces the rejection rate by parents and reduces the loss of data from fussy or uncooperative infants (Bechtoldt \& Hutz, 1979). Fantz, Fagan, and Kiranda (1975) have suggested that testing infants at home is more appropriate than testing in a laboratory when repeated testing is needed. The infants were tested repeatedly, using direction of gaze as the criterion measure until the subjects reached for a real object or they were 24 weeks of age; at that time, the prediction each subject would reach for a virtual image was evaluated.

\section{METHOD}

\section{Subjects}

Twenty-four infants, 14 males and 10 females, were tested approximately every 2 weeks until they either started reaching or were 24 weeks old. Six groups were defined by age at first test (i.e., the groups consisted of four 8-week-olds, six 9-weekolds, five 10-week-olds, and three subjects each in the three ages of 11,12 , and 13 weeks).

Parents were paid $\$ 2$ for each session. Names were obtained from birth announcements in a local newspaper.

The infants were usually fed and were wide awake at the time of the testing. Parents were asked about prior illnesses of the infant and gestational period. Most babies were born fullterm and had no significant medical history.

Only one subject was replaced; one of the 9-week-old females died at 16 weeks of age. Originally, only four babies were tested at the youngest age of 8 weeks. The results obtained in this group led us to test two more infants at this age. For these two additional babies, only one testing session was conducted.

\section{Apparatus}

The portable aluminum shadow-caster (see Gregory, 1964; Lee, 1969) used in the present study was designed to allow for the simultaneous projection of two images, one in each leftright half of the screen, with each illuminated area about $11 \mathrm{~cm}$ in diameter. The light sources in each half were two pairs of GE $25-W$ screw-based bulbs mounted in cylindrical reflectors, $38 \mathrm{~cm}$ from a plastic screen.

The shadows of a hollow brass cube, $52 \mathrm{~mm}$ on a side, appeared in each half of the screen; the cubes were located behind the screen, $30 \mathrm{~cm}$ from the light sources. All sides of both cubes were enlarged to about $5 \mathrm{~mm}$ with black tape to insure detection by the younger subjects. The cubes could be independently rotated around a horizontal axis, with varying speeds, making it possible to project the image of a rotating cube in one side of the screen and a stationary cube in the other.

Either one pair of Polaroid filters oriented $90 \mathrm{deg}$ apart or one pair of neutral-density filters could readily be inserted in front of each pair of light sources in such a way that it was possible to project the polarized image of the cube in either side or in both sides simultaneously. The interior walls were painted black, and the side walls were covered with black velvet; two baffles about $4 \mathrm{~cm}$ in width on the side walls kept wall reflections from the screen. Both displays had the same brightness value $(1.5 \mathrm{fc})$ either when the Polaroid or neutral-density filters were in front of the lights.

A tube $3.5 \mathrm{~cm}$ in diameter, extended from the screen to $12 \mathrm{~cm}$ to the rear of the box and centered in the middle of the screen, allowed an observer to watch the infant's eyes from behind the shadow-caster. The distance from the subject's face to the screen was about $40 \mathrm{~cm}$. Two buttons, one on each side of the shadow-caster, controlled the pens of a Rustrak event recorder provided for the recording on sensitive paper of the direction and duration of the subject's gaze.

\section{Procedure}

All infants were tested in their homes at a time when, according to the parents, they were usually awake and recently fed. Before the first session began, the procedure was explained to the parents, informed consent was obtained, and one parent answered questions about the medical history of the baby.

A session consisted of a series of five trials, ideally 30 -sec trials with a 5-sec intertrial interval, with the infant in a padded car seat. Because the main datum of interest in this study was the relative time the infant attended to each display, trials were extended if needed to make sure that $30 \mathrm{sec}$ of looking time was obtained for each subject. ${ }^{1}$

The first trial consisted of the presentation of the stationary stimulus, with the polarizers in front of the light sources on both sides. This first trial indicated any side preference of the subject.

The second trial, as the first test trial, consisted of the introduction of movement on the nonpreferred side. When no preference was shown, movement was first introduced on the baby's left side. Trial 3 was identical to Trial 2, with the cue of movement on the opposite (initially preferred) side. During both movement trials, the polarizers remained in front of the light beams; the cube on the appropriate side was oscillated about 180 deg back and forth at about .5 rps.

In Trial 4 the polarizers were changed to neutral-density filters on the nonpreferred side, as defined in Trial 1. The choice being offered to the baby, from an adult's point of view, was between the double shadows of a cube with no depth on the nonpreferred side and the single image of a cube that appeared to be a few centimeters out in front of the screen on the preferred side. The double shadows of the no-depth stimulus were also a novel and more complex stimulus (it has twice as many lines as the depth stimulus if fusion is achieved in the binocular retinal disparity or depth condition).

Trial 5 was identical to Trial 4 with the polarizers and neutraldensity filters changed to the other side. No movement cues were used in Trials 4 and 5.

The infants had the polarized glasses before their eyes during all five trials; one experimenter held the glasses precisely in front of the infant's eyes. The procedure, with the experimenter holding the glasses, was adopted because other methods tended to cause the glasses to slip as the infant moved around in the car seat. 
The motor development of the infants was informally observed by the experimenters, and when it seemed that a baby was ready to reach, a tentative decision was made to elicit reaching responses to colored objects and to cubes. When reaching responses could be obtained, usually by the 22 nd week, the infant was tested using the procedures and shadow-caster described by Bechtoldt and Hutz (1979). If the baby would not reach for real objects by the 24 th week, the testing was discontinued.

\section{Data Reduction}

Three distinct measures were obtained from the tapes in the event recorder: the time the infants looked at the display to their right, the time they looked at the display to their left, and the total length of the trial. The latter is the sum of the two former measures (total looking time) plus the time the infants did not look at the screen during the trial. These raw measures were converted into percentages of looking to the right and looking to the left.

The percentages of time looking to movement during Trials 2 and 3 and to the no-depth (nondisparity) stimulus during Trials 4 and 5 were averaged. If a subject watched the displays randomly or consistently fixated one side of the screen, he would get a score of about $50 \%$, which is the chance level of the present study.

Percentages were used for plotting graphs; analyses were carried out on arcsin transformations of the percentages.

\section{RESULTS}

\section{Stimulus Preference}

On Trials 4 and 5 the subjects could watch either the display with disparity or the display without disparity. With almost no exception, the subjects, at all age levels, preferred the display with no disparity. In only 2 instances out of $140(1.43 \%)$ was the stimulus disparity preferred. Therefore, the data analyzed on Trials 4 and 5 were the preferences for the no-depth stimulus.

\section{First Test}

The results obtained with each of the six age groups of infants in the movement and in the no-depth stimulus conditions are illustrated in Figure 1. The viewing times for both conditions are well above the chance level of $50 \%$. Only one significant effect was found between conditions; the viewing times percentage for movement was higher than that for the no-depth stimulus $[F(1,20)=4.43, \mathrm{p}<.05]$.

\section{Successive Testing}

The data for all age groups are combined in Figure 2 for the interval of 14-22 weeks. In order to establish whether successive testing has an effect on the infants' performance, an analysis was performed on the data from the first four sessions for each age group. Only the first four sessions were analyzed because some of the older subjects started reaching after the fourth session.

For the four sessions, only the difference in looking times between the movement and disparity trials was significant $[F(1,26)=31.67, \mathrm{p}<.001]$.

During the first four sessions, mean percent viewing times to the movement stimuli for all ages combined

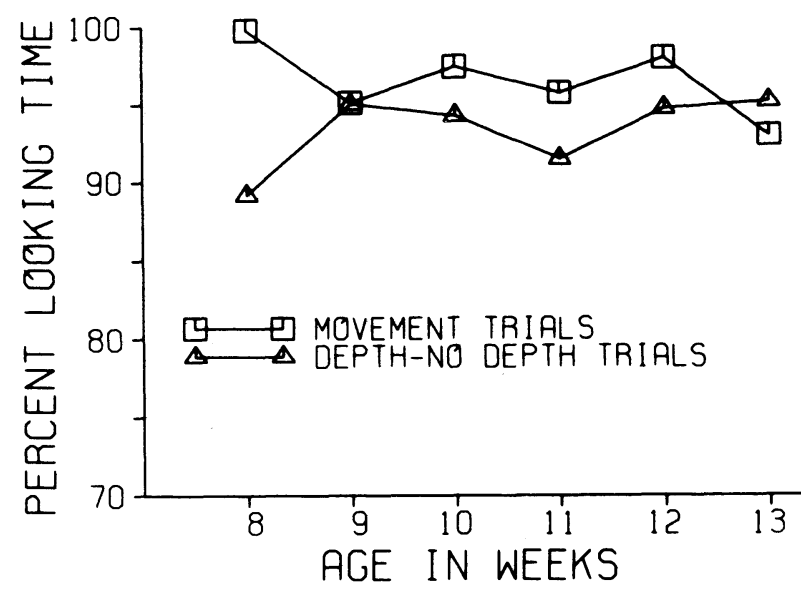

Figure 1. Percent looking time for movement and depth/ no-depth stimuli in first testing session.

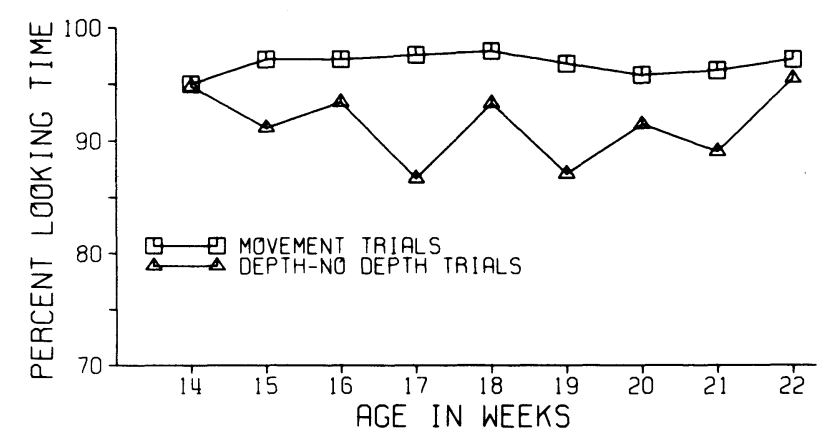

Figure 2. Percent looking time for movement and depth/ no-depth stimuli for all cases combined at ages 14 to 22 weeks.

were $96.79 \%, 95.98 \%, 97.41 \%$, and $94.19 \%$, for Sessions 14 , respectively. Mean percent viewing times to the no-depth stimulus from Sessions $1-4$ for all cases combined were $92.23 \%, 93.78 \%, 93.54 \%$, and $90.47 \%$, respectively. The time span between sessions was 2 weeks for most subjects; for a few subjects the tests were 1 or 3 weeks apart.

\section{Reaching Responses}

Of the 24 subjects, $20(83 \%)$ reached consistently for the virtual image. Disregarding the one case of the infant who did not reach for real objects, the proportion of reaching subjects was $87 \%$. One infant who had not reached at 24 weeks of age still did not reach for the virtual image 1 week later. The relative frequency of $83 \%$ is comparable with the proportion of $71 \%$ obtained with an earlier sample (Bechtoldt \& Hutz, 1979); the $95 \%$ confidence interval for the $83 \%$ value is .61 to .94 , a range excluding the chance value of $50 \%$

\section{DISCUSSION}

The results very clearly indicate that 8 -week-old infants can indeed make binocular discriminations. With one exception, all infants in the sample discriminated between the displays in the first session by showing a strong preference for the no-depth 
stimulus. One infant, an 8-week-old female, performed at chance level. ${ }^{2}$ Evidence for binocular discrimination was found in virtually every session in the testing of 24 subjects in the sample.

Perhaps the most surprising result was the strength of the preference for the no-depth stimulus in the disparity vs. nondisparity trials. There are at least two possible explanations for the no-depth preference. This preference may be associated either with novelty, if habituation has taken place, or with the increase in complexity, or with both. If the infants have stereopsis, they saw a cube a few centimeters out in front of the screen, either rotating or stationary on three trials. If they did not have stereopsis but were nevertheless able to fuse the image, they saw a flat image of a cube (like some adults with low stereoacuity). If they saw the cube in depth or fused, then when the neutral-density filters replace the polarizers in Trials 4 and 5 a new and more complex stimulus is presented, complexity here being defined as the apparent number of lines in the stimulus. In any case, preference for a given display, if consistent, is a good indicator of the ability to discriminate. If the infants could not fuse the images, there would be neither novelty nor greater complexity in Trials 4 and 5 to serve as a basis for discrimination.

The data obtained from the fixation-of-gaze observations led to the prediction that all 24 subjects tested longitudinally should already have stereopsis when the motor skills required for a reaching response were developed; the observation of $83 \%$ reaching is consistent with this prediction. The nonreaching behavior of three infants is not necessarily an indication of lack of binocular functioning; these infants did not appear to pay much attention to the display at the time of testing.

In one case only, an infant 22 weeks of age exhibited a startle response when the cube appeared to be in a collision course with his face on the last trial of the reaching test procedure.

\section{REFERENCE NOTE}

1. Held, R. Personal communication, March 13, 1980.

\section{REFERENCES}

Appel, M. A., \& Campos, J. J. Binocular disparity as a discriminable stimulus parameter for young infants. Journal of Experimental Child Psychology, 1977, 23, 57-77.

Aslin, R. N. Development of binocular fixation in human infants. Journal of Experimental Child Psychology, 1977, 23, 133-150.

Atrinson, J., \& Braddick, O. Stereoscopic discrimination in infants. Perception, 1976, 5, 29-38.

Bechtoldt, H. P., \& Hutz, C. S. Stereopsis in young infants. Journal of Pediatric Ophthalmology and Strabismus, 1979, 16, 49-52.

Benton, A. L., \& Hecaen, H. Stereoscopic vision in patients with unilateral cerebral disease. Neurology, 1970, 20, 1084-1088.

Bower, T. G. R., Broughton, J. M., \& Moore, M. K. The coordination of visual and tactual input in infants. Perception \& Psychophysics, 1970, 8, 51-53.

Carmen, A., \& Bechtoldt, H. P. Dominance of the right cerebral hemisphere for stereopsis. Neuropsychologia, 1969, 7, 29-39.

Dobson, V., \& Teller, D. Y. Visual acuity in human infants: A review and comparison of behavioral and electrophysiological studies. Vision Research, 1978, 18, 1469-1483.

FANTZ, R. L. A method for studying early visual development. Perceptual and Motor Skills, 1956, 6, 13-15.

Fantz, R. L. Pattern vision in young infants. Psychological Record, 1958, 8, 43-47.
Fantz, R. L., Fagan, J. F., III, \& Kiranda, S. B. Early visual selectivity. In L. B. Cohen \& P. Salapatek (Eds.), Infant perception: From sensation to cognition (Vol. 1). New York: Academic Press, 1975.

Fox, R., Aslin, R. N., Shea, S. L., \& Dumais, S. T. Stereopsis in human infants. Science, 1980, 207, 323-324.

Giviazda, J., Brill, S., \& Held, R. New methods for testing infant vision. Sightsaving Review, 1979, 49, 61-69.

Gordon, F. R., \& Yonas, A. Sensitivity to binocular depth information in infants. Journal of Experimental Child Psychology, 1976, 22, 413-422.

Gregory, R. L. Stereoscopic shadow-images. Nature, 1964, 203, 1407-1408.

HAMSHER, $\mathbf{K}$. Stereopsis and unilateral brain disease. Investigative Opthalmology and Visual Science, 1978, 17, 336-343.

HELD, R. Development of visual resolution. Canadian Journal of Psychology, 1979, 33, 213-221.

LEE, D. L. Theory of the stereoscopic shadow-caster: An instrument for the study of binocular kinetic space perception. Vision Research, 1969, 9, 145-146.

ReESE, H. W., \& LiPSITT, L. P. Experimental child psychology. New York: Academic Press, 1970.

RICHARDS, W. Stereopsis with and without monocular contours. Vision Research, 1977, 17, 967-969.

Slater, A. M., \& Findlay, J. M. Binocular fixation in the newborn baby. Journal of Experimental Child Psychology, 1975, 20, 248-273.

Yonas, A., Oberg, C., \& Norcia, A. Development of sensitivity to binocular information for the approach of an object. Developmental Psychology, 1978, 14, 147-152.

Yonas, A., \& Pick, H. L. An approach to the study of infant space perception. In L. B. Cohen \& P. Salapatek (Eds.), Infant perception: From sensation to cognition (Vol. 2). New York: Academic Press, 1975.

\section{NOTES}

1. In order to insure that meaningful data would be collected, a series of special procedures was devised for handling subjects. Pacifiers were used whenever the babies would take them. Some infants were even fed from a bottle when they were watching the displays. In some cases, we used the infant's own car seat, or the subject would sit in the mother's lap while she was seated on the floor. As a general rule, we would do whatever was needed to keep the infants calm and their attention directed to the displays, subject to the restriction that the procedure that was used would not bias the data.

2. This infant watched the novel stimulus $51.53 \%$ of the time, indicating no discrimination. According to the mother's report, when she was about 16 weeks of age a pediatrician suspected that she might have strabismus; subsequently, an ophthalmologist found a small esodeviation of the left eye. A retest with the preference procedure when she was 19 weeks old showed a failure to discriminate; she looked at the novel stimulus $50.12 \%$ and $52.41 \%$ of the time in two separate sessions. When reaching for real objects was well established at 23 weeks of age, she was tested with the shadow-caster; however, no reaching for the virtual image was seen. It seems that the only failure to discriminate observed can be explained in terms of lack of binocular vision.

(Received for publication June 2, 1980.) 